\title{
Solitary coronary artery: An unusual cause of atypical chest pain
}

\author{
Laxman Dubey ${ }^{1}$, Ridhi Adhikari², Suresh Deep ${ }^{3}$ \\ ${ }^{1}$ Cardiology Division, Crimson Hospital, Tilottama, Nepal \\ ${ }^{2}$ Radiology Division, Butwal Hospital Pvt Ltd, Butwal, Nepal \\ ${ }^{3}$ Medicine Department, Chitwan Medical College, Bharatpur, Nepal \\ Corresponding Author: Laxman Dubey \\ Cardiology Division, Crimson Hospital, Tilottama-5, Rupandehi, Nepal \\ Email: dr.laxmandubey@gmail.com
}

Cite this article as: Dubey L, Adhikari R, Deep S. Solitary coronary artery: An unusual cause of atypical chest pain. Nepalese Heart Journal 2019; Vol 16(1), 55-56

Received date: 12 th February 2019

Accepted date: 30th March 2019

\begin{abstract}
Coronary arteries arising from single coronary sinus is a rare congenital anomaly. We report a 30 -year-old male who presented with atypical chest pain and computed tomography coronary angiography revealed a solitary coronary artery originating from a single ostium in the right sinus of Valsalva.
\end{abstract}

Keywords: Anomalous origin; Atypical chest pain; CT angiography; Solitary coronary artery.

DOI: https://doi.org/10.3126/njh.v16i1.23903

\section{Introduction}

Solitary coronary artery is a coronary artery anomaly that describes the origin of both right coronary artery (RCA) and left coronary artery (LCA) from a single aortic sinus. It is usually diagnosed incidentally during coronary artery angiograms or on postmortem evaluations. ${ }^{1}$ A single coronary artery arising from one aortic sinus is an extremely rare congenital anomaly that is seen in only $0.0024 \%-0.044 \%$ of the population..$^{2,3}$ Of these, a single coronary artery arising from the right sinus of Valsalva represents one of the rarest forms of all coronary anomalies. We report a case of a single coronary artery arising from the right coronary sinus of Valsalva, in a 30-year-old man who presented with atypical chest pain.

\section{Case Report}

A 30-year-old male presented with recurrent left sided chest pain that was non-radiating. He was a smoker with no history of hypertension, diabetes mellitus or family history of coronary heart disease. He was not under any medications. His blood pressure was $120 / 80 \mathrm{mmHg}$, pulse rate of 85 beats per minute. Electrocardiography showed sinus rhythm with $\mathrm{J}$ point elevation in leads V4-V6 suggestive of lateral wall benign early repolarization pattern. Transthoracic echocardiography showed normal left ventricular systolic function and no regional wall motion abnormality. Exercise treadmill test was negative for inducible myocardial ischemia. Since he had several episodes of chest pain, multislice computed tomography (MSCT) coronary angiography was performed to rule out coronary artery disease. In MSCT coronary angiography right anterior oblique (Fig. 1a)

@Nepalese Heart Journal. Nepalese Heart Journal retains copyright and work is simultaneously licensed under Creative Commons Attribution License CC - BY 4.0 that allows others to share the work with an acknowledgement of the work's authorship and initial publication in this journal. 
and left anterior oblique (Fig. 1b) views we saw a single coronary artery arising from the right sinus of Valsalva trifurcating into RCA, left anterior descending (LAD) artery and left circumflex $(\mathrm{LCx})$ artery. There was no coronary artery ostium at the left sinus of Valsalva. LAD coursing from its origin in right side towards the left side in the anterior inter-ventricular groove and the LCx in the left atrio-ventricular groove. The common trunk was 5.6 $\mathrm{mm}$ in diameter and $7.2 \mathrm{~mm}$ in length. The LAD and LCx had prepulmonic course (Lipton type R-IIA). ${ }^{4}$ The coronary arteries were free of atherosclerotic disease. We advised the patient to quit smoking and to avoid competitive sports and strenuous exercise.

\section{Figure Legend}

Figure: Multislice computed tomography (MSCT) coronary angiography

Right anterior oblique (Fig. 1a) and left anterior oblique (Fig. 1b) views show a solitary coronary artery arising from the right sinus of Valsalva trifurcating into right coronary artery (RCA), left anterior descending (LAD) artery and left circumflex (LCx) artery.

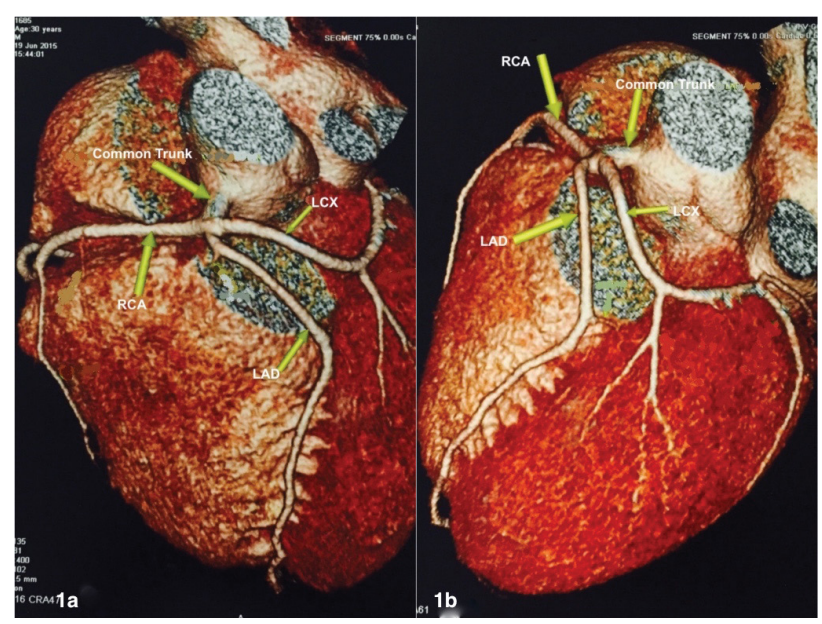

\section{Discussion}

Solitary coronary artery is a rare congenital anomaly that is usually diagnosed incidentally. In a large series of 126,595 patients undergoing coronary angiography, a single coronary artery from the right sinus of Valsalva was found in $0.019 \%{ }^{3}$

Several classification systems for coronary artery abnormalities exist. Lipton et $\mathrm{al}^{4}$ classified the coronary variations according to the origin and anatomical course relating to the ascending aorta and pulmonary trunk. According to origin type L represents the single coronary artery originates from the left sinus and type $\mathrm{R}$ indicates that the single coronary artery originates from the right sinus. According to anatomical course these types are then classified as I to III. Class I follows the anatomical course of either an RCA or LCA. Class II indicates one coronary artery arising from the proximal part of the normally located opposite coronary artery. In class III, the LAD and LCx arise separately from the proximal part of a normal RCA. Classes II and III are then designated as anterior (type A) or posterior (type $\mathrm{P}$ ) according to their route in relation to the pulmonary artery, or interarterial (type B) if it courses between the ascending aorta and pulmonary trunk.
The solitary coronary artery anomaly is usually asymptomatic, but may present as myocardial ischemia, syncope or sudden cardiac death during exercise depending on its course and the presence and severity of atherosclerosis. ${ }^{1}$ The actual prognosis of patients with single coronary artery is not clear and a treatment strategy guide of this condition does not exist yet. Revascularization is recommended only if anomalous coronary artery courses between aorta and pulmonary artery or there is significant atherosclerotic disease. Due to the risk of sudden death in patients whose anomalous coronary artery courses between aorta and pulmonary artery, coronary artery bypass surgery is useful even if patients have not severe atherosclerotic coronary stenosis. ${ }^{1}$ In our patient, we assumed that the cause for his atypical chest pain could be due to the presence of a solitary coronary artery. It has been reported that $15 \%$ of isolated solitary coronary artery may have myocardial ischemia directly caused by the abnormal anatomy of the arteries and not by coronary artery disease. ${ }^{5}$ In such cases MSCT coronary angiography can be done to rule out coronary artery disease. Moreover, MSCT coronary angiography is a non invasive, also a $3 \mathrm{D}$ imaging technique may be useful to evaluate the coronary anatomy and define the coronary artery anomalies. ${ }^{6}$

\section{Conclusion}

A single coronary artery arising from the right sinus of Valsalva is extremely rare and usually asymptomatic. However, some may present with chest pain, syncope, heart failure and sudden death. The coronary anomaly reported upon represent rare Lipton type R-IIA subtype.

Conflict of Interest: None

Funding: None

\section{References}

1. Ozyurtlu F, Acet H, Bilik MZ, et al. A rare coronary artery anomaly: single coronary artery originate from right sinus Valsalva R-IIP sub-group type. Cardiol Res 2012;3:140-42 https://doi.org/10.4021/cr185w

2. Desmet W, Vanhaecke J, Vrolix M, et al. Isolated single coronary artery: a review of 50000 consecutive coronary angiographies. Eur Heart J 1992;13:1637-40 https://doi.org/10.1093/oxfordjournals.eurheartj.a060117

3. Yamanaka O, Hobbs RE. Coronary artery anomalies in 126,595 patients undergoing coronary arteriography. Cathet Cardiovasc Diagn 1990;21:28-40 https://doi.org/10.1002/ccd.1810210110

4. Lipton MJ, Barry WH, Obrez I, et al. Isolated single coronary artery: diagnosis, angiographic classification, and clinical significance. Radiology 1979;130:39-47 https://doi.org/10.1148/130.1.39

5. Muhyieddeen K, Polsani VR, Chang SM. Single right coronary artery with apical ischemia. Eur Heart J Cardiovasc Imaging 2012;13:533 https://doi.org/10.1093/ehjci/jes013

6. Eyuboglu M, Cuce F. Single coronary artery accompanying myocardial bridging on LAD and retroaortic course of LCX. Int J Cardiovasc Acad 2015;1:53-55 https://doi.org/10.1016/j.ijcac.2015.09.001 